\title{
SEISMO-MAGNETIC FIELD ANOMALIES DETECTION USING SWARM SATELLITES (ALPHA, BRAVO AND CHARLIE)
}

\author{
Mehdi Akhoondzadeh * \\ Remote Sensing Division, School of Surveying and Geospatial Engineering, College of Engineering, \\ University of Tehran, Iran- makhonz@ut.ac.ir
}

Commission VI, WG VI/4

KEY WORDS: Earthquake, Swarm satellites, Ionosphere, Magnetic field.

\begin{abstract}
:
Among the lithospheric, atmospheric and ionospheric earthquake precursors, it seems that the ionospheric anomalies showing a meaningful association with seismic activities during absence of solar and geomagnetic activities. Unfortunately there are a limited number of satellite sensors to survey the ionosphere and study on seismo-ionospheric anomalies. This paper represents the data analysis results of Swarm satellites including Alpha, Bravo and Charlie data around the Mexico (September 8, 2017) earthquake. The orbital analysis and time series of magnetic field parameters (magnetic scalar and vectors $(\mathrm{X}, \mathrm{Y}, \mathrm{Z}$ ) components) inside the Dobrovolsky's area show anomalous variations close to the time and locations of the Mexico earthquake. There is a concavity or convexity variations in the some of the time-series to the centre of the earthquake day. In other words, from about 90 days before the event a decreasing or increasing trend in variations of parameters is observed and exactly after the earthquake day its trend changes. It should be noted that the variations of the solar and geomagnetic indices must indicate a normal behaviour during the observed seismo-ionospheric anomalies. Therefore this study indicates that the Swarm satellites measurements play an undeniable role in progress the studies of the ionospheric precursors.
\end{abstract}

\section{INTRODUCTION}

There are many scientific evidences implying on observation of seismic LAI (Lithospheric-Atmospheric-Ionospheric) anomalies which begins a few days before the earthquake and stay until a few days after it (Parrot, 1995; Hayakawa and Molchanov, 2002; Pulinets and Boyarchuk, 2004). Up to now, several earthquake mechanisms have been proposed to explain the occurrence process of the earthquake precursors, but this topic is still a challenging task (Molchanov and Hayakawa, 2008; Feund, 2011; Pulinets and Ouzounov, 2011). Satellite measurements due to their wide coverage, being up to date, free access are a complementary data in addition to the ground stations measurements to study on earthquake precursors.

Swarm is an ESA (European Space Agency) satellite mission of three satellites to survey precisely the geomagnetic signals from earth's core, mantle, crust and oceans, as well as the ionosphere and magnetosphere. The three swarm satellites are named $\mathrm{A}$ (Alpha), B (Bravo) and C (Charlie). The two satellites of A and $\mathrm{C}$ are flying almost side-by-side with longitude separation of $1.4^{\circ}$ at equator, an altitude close to $450 \mathrm{~km}$, and inclination of 87.4 . The polar orbit altitude of the third satellite (b) is close to $510 \mathrm{~km}$. This specific constellation allows the scientists to observe the small space-scale variations of the geomagnetic field, particularly those linked to the lithospheric field (De Santis et al., 2017). The main mission sensors are a couple of magnetometers, i.e. an absolute scalar magnetometer (ASM) providing, in nominal mode, measurements of the field intensity and a vector field magnetometer (VFM) mounted halfway along the boom on an optical bench together with the star trackers, providing field directions. Other sensors complete the payload of each satellite, in particular, two electric field and particle sensors (Langmuir probes), a GPS antenna and an accelerometer (De Santis et al., 2017). In contrast with the previous magnetic satellites, swarm measurements are made at different orbits and altitudes. Therefore these constellation and orbitography could be lead to create a precise investigator of geomagnetic field to detect the anomalies likely connected to earthquakes in preparation phase. De Santis et al. (2017) suggest that the potential LAI (Lithosphere Atmosphere Ionosphere) coupling would extend over a rather large interval of around a month before and after the 2015 Nepal main-shock using Swarm satellite data analysis. Akhoondzadeh et al. (2017) did a multi-precursor's analysis and then using absolute scalar magnetometer, vector field magnetometer and electric field instrument on board Swarm satellites and GPS (Global Positioning System) measurements the variations of the electron density and temperature, magnetic field and TEC (Total Electron Content) have been investigated to find the potential seismic anomalies around the strong Ecuador $\left(\mathrm{M}_{\mathrm{w}}=7.8\right)$ earthquake of 16 April 2016.

\section{METHODOLOGY}

Absolute scalar magnetometer measures the strength of the magnetic field to calibrate the vector field magnetometer. Vector field magnetometer makes high precision measurements

\footnotetext{
* Corresponding author
} 
of the magnitude and direction of the magnetic field, i.e. the field's vector. In order to detect the potential anomalies in the variations of the magnetic field, at the first step, all tracks of each satellite based on the local time were divided to two classes of the daytime and the nighttime. Then for each sample of a track, the difference between the measured magnetic field value and the predicted magnetic field value using the IGRF (International Geomagnetic Reference Field) model was calculated. At the next step, the median of the residuals of magnetic values of the daytime and nighttime tracks was obtained and the time-series of the magnetic field median values during the studied were constructed. To eliminate the nonlinear variations a polynomial of 3 degree was fitted to the time series and the residuals values were calculated. . If the residual value exceeds the pre-defined threshold value (i.e. $M \pm 1.25 \times I Q R ; M$ and $I Q R$ are the median and the inter-quartile range parameters, respectively), the observed parameter in geomagnetically quiet conditions $\left.\left(|\mathrm{Dst}| \leq 20 \mathrm{nT}, \mathrm{a}_{\mathrm{p}}<10 \mathrm{nT}\right)\right)$ is regarded as a candidate of a seismic anomaly.

\section{RESULTS}

In Mexico $\left(15.022^{\circ} \mathrm{n}, 93.899^{\circ} \mathrm{w}, 47.40 \mathrm{~km}\right.$ depth) a strong earthquake of $\mathrm{M}_{\mathrm{w}}=8.2$ happened at 04:49:19 UTC on September 8, 2017. Figure 1 illustrates the daytime scalar magnetic field values measured using satellite $\mathrm{A}$ at during the studied period. A dominant anomaly is seen on earthquake day. It is seen that there is a convex curve to the centre of the earthquake day (the black arrows in figure 1). There is a decreasing trend from about 103 days before event and immediately after the shock it increases. Therefore it can be considered as a long term precursor.

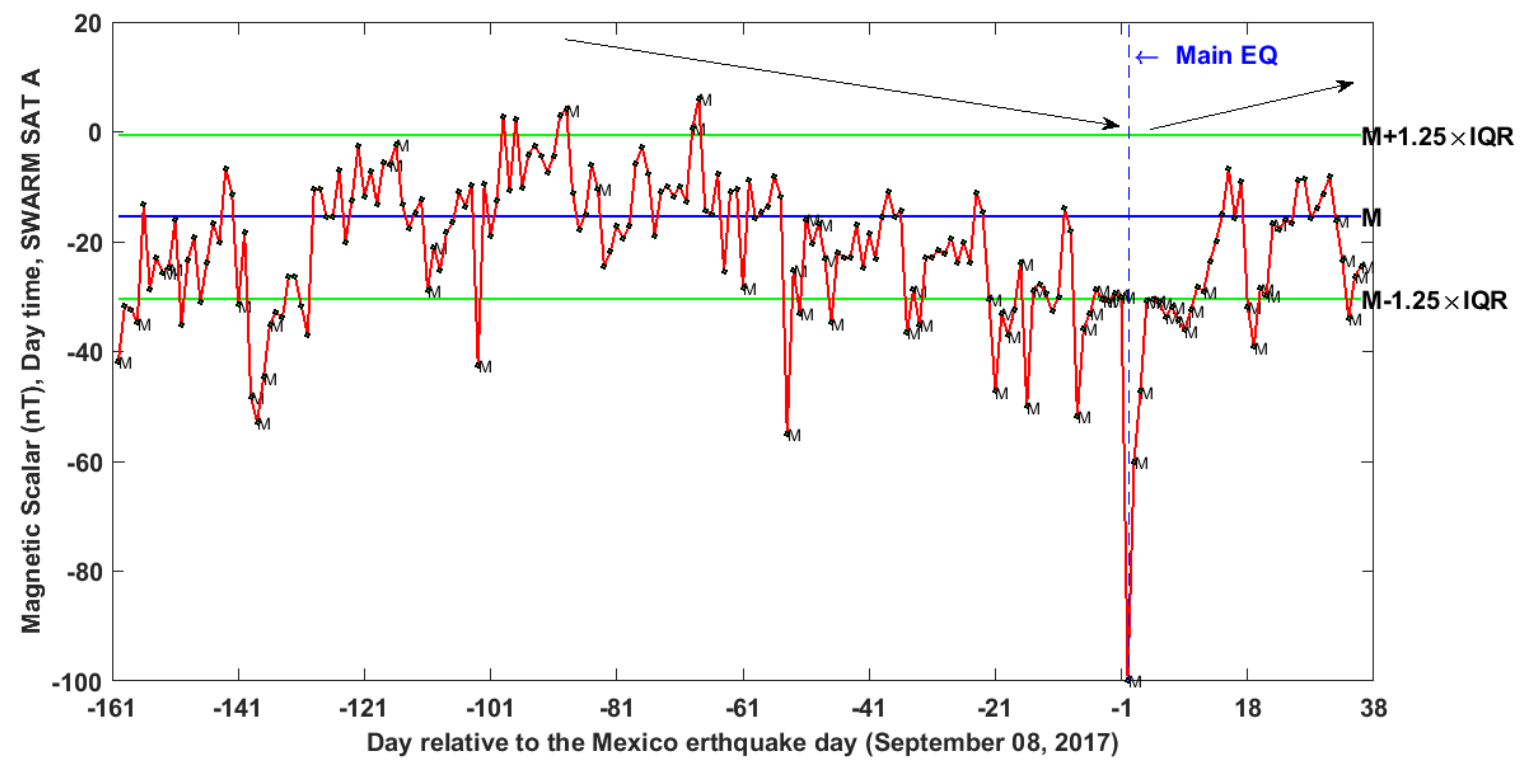

Fig. 1. Results of Swarm A scalar magnetic field data analysis for the Mexico earthquake (08 September 2017) from 01 April to 15 October 2017. The earthquake day is represented as vertical line. The green horizontal lines indicate the upper and lower bounds $(M \pm 1.25 \times I Q R)$. The blue horizontal line indicates the median value $(M)$. The values of the median and the allowable bounds were calculated only using the quiet geomagnetic days. The $\mathrm{x}$-axis represents the day relative to the earthquake day. Theyaxis represents the daytime scalar magnetic field median values for each day.

Figure 2 shows the night time corrected magnetic field vector $Y$ values measured using satellite $\mathrm{A}$ at during the period of

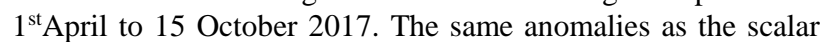
magnetic field anomalies are observed and the similar conclusions can be achieved about the seismic nature of them. Figure 3 illustrates night time corrected magnetic field vector $\mathrm{Y}$ values measured using satellite $\mathrm{C}$ during the period of $1^{\text {st }}$ April to 15 October 2017 . There are a striking anomaly on earthquake day and also a convex variations to the centre of the earthquake day, similar to the observed anomalies in the similar parameter measured using Swarm A satellite.

Figure 4 (a) illustrates the recorded track of Swarm A satellite close to the Mexico earthquake epicenter on 08 September, 2017. The earthquake epicentre, track and Dobrovolsky's area are shown as a red asterisk, a red line and a green circle, respectively. The track crossed the Dobrovolsky's area between the 04:21:41 and 04:39:54 UTC. The horizontal and vertical axes represent the geographic longitude and latitude coordinates, respectively. The differences between the timeseries of the first derivatives of the measured magnetic fields scalar and vectors $(\mathrm{X}, \mathrm{Y}, \mathrm{Z})$ values and a fitted polynomial of degree 12 along this track are shown in Figures 4 (b, c, d and e) respectively. The vertical axis represents the geomagnetic latitude. Every four time series of the residual curves of the scalar and vector components show anomalous variations around the earthquake location (the red arrows in Figure 4). 


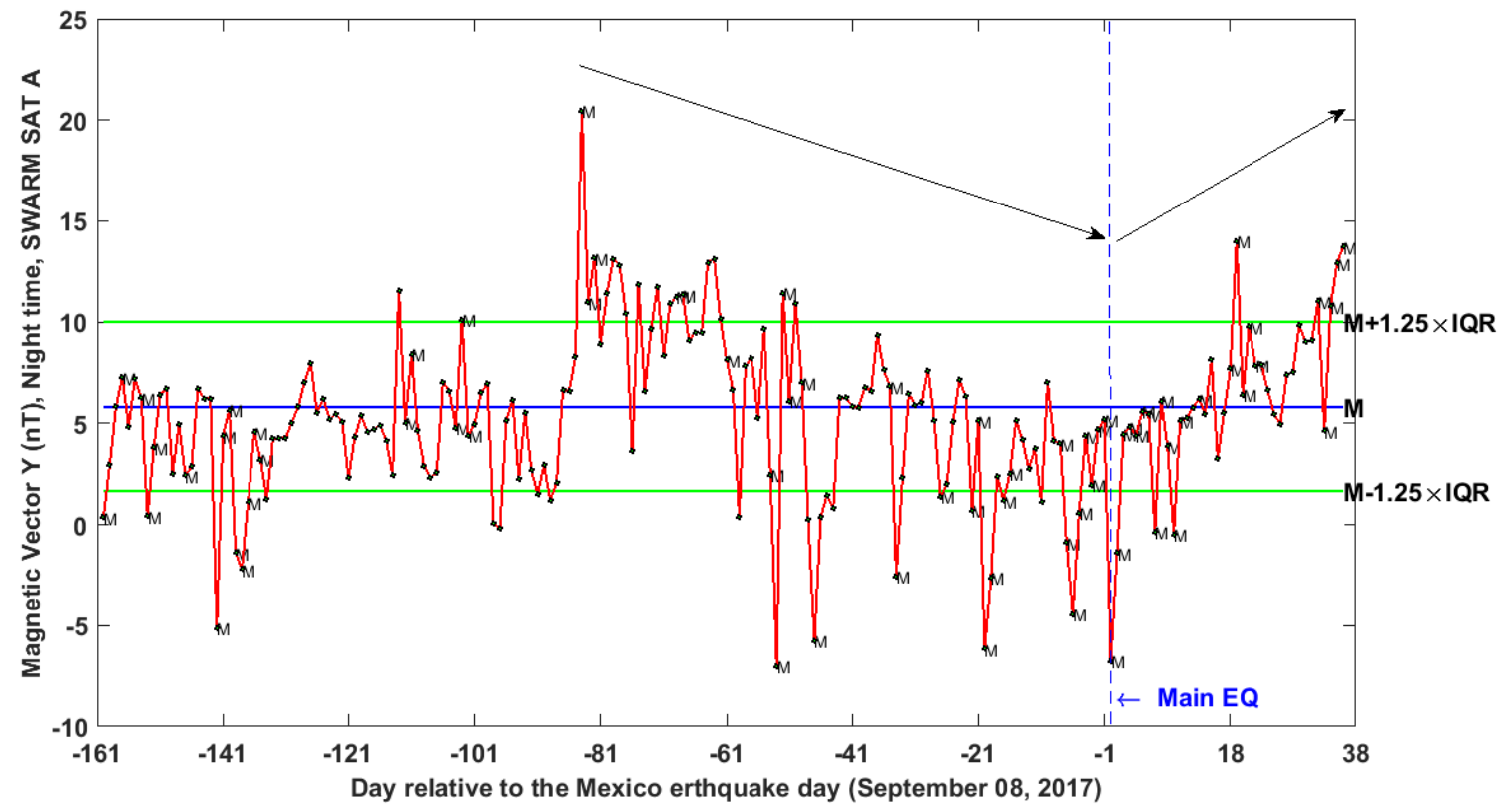

Fig. 2. Same as Fig. 1 but the y-axis represents the night time vector Y magnetic field median values for each day.

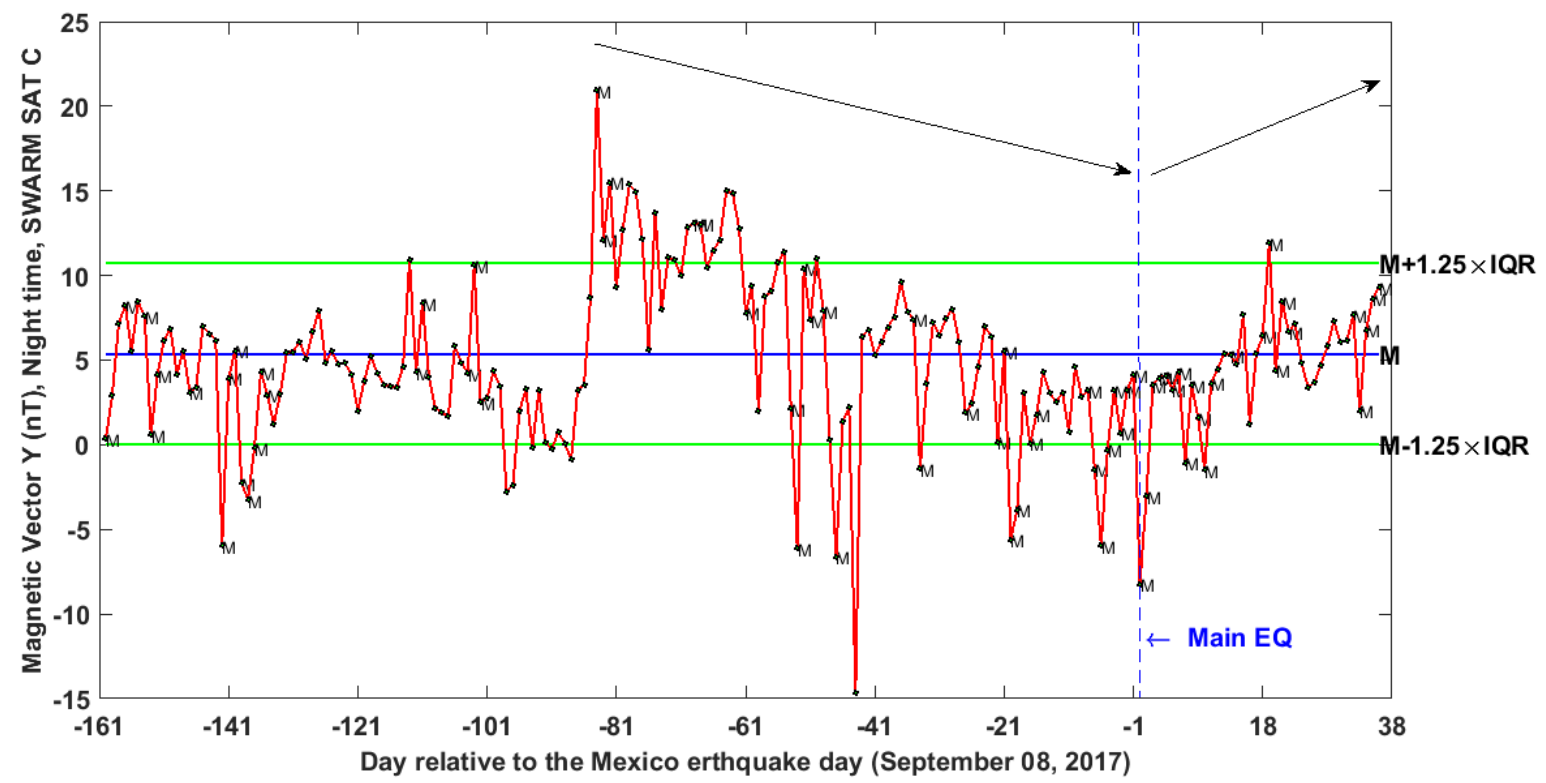

Fig. 3. Same as Fig. 2 but the y-axis represents the night time vector $Y$ magnetic field median values for each day measured by Swarm C. 


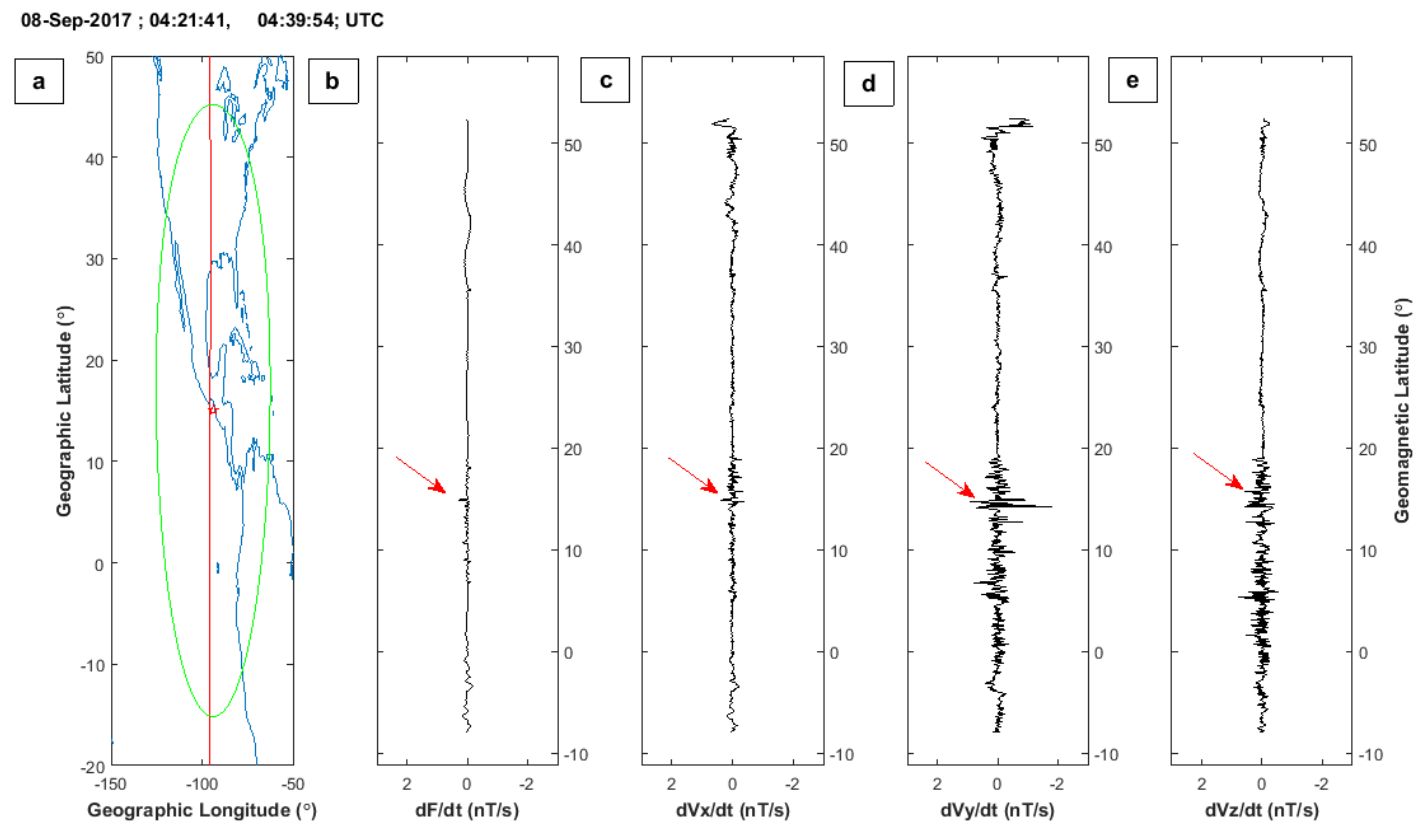

Fig. 4. Results of Swarm A track analysis for the Mexico earthquake (08 September 2017) on earthquake day. (a) The earthquake epicenter, the track and Dobrovolsky's area are shown as a red asterisk, a red line and a green circle, respectively. The track passed the Dobrovolsky's area between the 04:21:41 and 04:39:54UTC. The horizontal and vertical axes represent the geographic longitude and latitude. (b), (c), (d) and (e) The differences between the time-series of the derivatives of the measured magnetic fields scalar and vectors $(\mathrm{X}, \mathrm{Y}, \mathrm{Z})$ values, respectively, and a fitted polynomial of 12 degree along this track. The vertical axis represents the geomagnetic latitude.

\section{CONCLUSIONS}

This paper attempts to acknowledge the capabilities of the Swarm satellites data to analyse the earthquake precursors. The constellation and orbitography of Swarm satellites are a key advantage that causes the Earth' ionosphere is surveyed precisely in appropriate time resolution. Since Swarm A and C are flying almost side-by-side with longitude separation of $1.4^{\circ}$ at equator and an altitude close to $450 \mathrm{~km}$, therefore their measurements can be used to confirm each other. The results of this paper indicate that almost all magnetic parameters measured by each satellite show a dominant anomaly on earthquake day. The investigation in tracks of all satellites crossing the Dobrovolsky's area close to earthquake time, confirms the observed clear anomaly on earthquake day. There is a concavity or convexity variations in the some of the timeseries to the center of the earthquake day. In other words, from about 90 days before the event a decreasing or increasing trend in variations of parameters is observed and exactly after the earthquake day its trend changes. Therefore the diversity of the ionospheric parameters measured by 3 Swarm satellites can play an important role in earthquake multi-precursors studies.

\section{ACKNOWLEDGEMENTS}

The author would like to acknowledge the ESA for the Swarm data and the NOAA for the geomagnetic indices. This work was done under supervision of the Iran National Science Foundation (INSF) and founded with No. 96008126.

\section{REFERENCES}

Akhoondzadeh, M., De Santis, A., Marchetti, D., Piscini, A., Cianchini, G., 2017. Multi precursors analysis associated with the powerful Ecuador $\left(\mathrm{M}_{\mathrm{w}}=7.8\right)$ earthquake of 16 April 2016 using Swarm satellites data in conjunction with other multiplatform satellite and ground data. Advances in Space Research, doi.org/10.1016/j.asr.2017.07.014.

Bilitza, D., Altadill, D., Truhlik, V., Shubin, V., Galkin, I., Reinisch, B., Huang, X., 2017. International reference ionosphere 2016: From ionospheric climate to real-time weather predictions. Space Weather, doi: 1002/2016SW001593.

De Santis, A., Balasis, G., Pavon-Carrasco, F. J., Cianchini, G., and Mandea, M., 2017. Potential earthquake precursory pattern from space: The 2015 Nepal event as seen by magnetic Swarm satellites. Earth and Planetary Science Letters., 461, 119-126.

Dobrovolsky, I. R., Zubkov, S. I., and Myachkin, V. I., 1979. Estimation of the size of earthquake preparation zones.Pure Appl. Geophys. 117, 1025-1044.

Freund, F.T., 2011. Pre-earthquake signals: underlying physical processes. J. Asian Earth Sci.41, 383-400. 
Guglielmi, A. V., Lavrov, I. P., Sobisevich, A. L., 2015. Storm sudden commencements and earthquakes. Solar-Terrestrial Physics, 1 (1), 98-103.

Haagmans, R., Bock, R., Rider, H., 2013. Swarm; ESA's magnetic field mission. www.ESA.int.

Molchanov, O. A., 2008. Hayakawa, M. Seismo electromagnetics and related phenomena: history and latest results, TERRAPUB, Tokyo, pp. 189.

Olsen, N., Friis-Christensen, E., Floberghagen, R., Alken, P., Beggan, C. D., Chulliat, A., Visser, P. N., 2013. The Swarm Satellite Constellation Application and Research Facility (SCARF) and Swarm data products. Earth, Planets and Space, 64(11), 1189-1200. DOI: 10.5047/eps.2013.07.001.

Parrot, M., 2006. First results of the DEMETER micro-satellite.Planet. Space Sci., 54(5), 411-557.

Píša, D., Parrot, M., Santolík, O. 2011. Ionospheric density variations recorded before the $2010 \mathrm{Mw} 8.8$ earthquake in Chile. JGR, 116, A08309.

Pulinets, S.A., Ouzounov, D., 2011. Lithosphere-AtmosphereIonosphere Coupling (LAIC) model: an unified concept for earthquake precursors validation. J. Asian Earth Sci.41, 371382 .

Tronin, A., Hayakawa, M., and Molchanov, O. A., 2002. Thermal IR satellite data application for earthquake research in Japan and China. J. Geodyn., 33, 519-534. 